\title{
Patterns of multimorbidity and their association with health outcomes within Yorkshire, England: baseline results from the Yorkshire Health Study
}

Jessica Li $\mathrm{i}^{1,4^{*}}$ D, Mark Green², Ben Kearns ${ }^{1}$, Eleanor Holding ${ }^{1}$, Christine Smith³ ${ }^{3}$ Annette Haywood ${ }^{1}$, Cindy Cooper ${ }^{1}$, Mark Strong ${ }^{1}$ and Clare Relton ${ }^{1}$

\begin{abstract}
Background: Multimorbidity is increasingly being recognized as a serious public health concern. Research into its determinants, prevalence, and management is needed and as the risk of experiencing multiple chronic conditions increases over time, attention should be given to investigating the development of multimorbidity through prospective cohort design studies. Here we examine the baseline patterns of multimorbidity and their association with health outcomes for residents in Yorkshire, England using data from the Yorkshire Health Study.

Methods: Baseline data from the Yorkshire Health Study (YHS) was collected from 27,806 patients recruited between 2010 and 2012. A two-stage sampling strategy was implemented which first involved recruiting 43 general practice surgeries and then having them consent to mailing invitations to their patients to complete postal or online questionnaires. The questionnaire collected information on chronic health conditions, demographics, health-related behaviours, healthcare and medication usage, and a range of other health related variables. Descriptive statistics (chi-square and t tests) were used to examine associations between these variables and multimorbidity.

Results: In the YHS cohort, 10,332 participants (37.2\%) reported having at least two or more long-term health conditions (multimorbidity). Older age, BMI and deprivation were all positively associated with multimorbidity. Nearly half (45.7\%) of participants from the most deprived areas experienced multimorbidity. Based on the weighted sample, average health-related quality of life decreased with the number of health conditions reported; the mean EQ-5D score for participants with no conditions was 0.945 compared to 0.355 for participants with five or more. The mean number of medications used for those without multimorbidity was 1.81 (range 1-13, SD =1.25) compared to 3.81 (range 1-14, $S D=2.44$ ) for those with at least two long-term conditions and 7.47 (range 1-37, SD = 7.47) for those with $5+$ conditions.

Conclusion: Patterns of multimorbidity within the Yorkshire Health Study support research on multimorbidity within previous observational cross-sectional studies. The YHS provides both a facility for participant recruitment to intervention trials, and a large population-based longitudinal cohort for observational research. It is planned to continue to record chronic conditions and other health related behaviours in future waves which will be useful for examining determinants and trends in chronic disease and multimorbidity.
\end{abstract}

Keywords: Multimorbidity, Multiple cohort trial, Health conditions, Long-term conditions

\footnotetext{
* Correspondence: jessica.li@sheffield.ac.uk

${ }^{1}$ School of Health and Related Research (SCHARR), University of Sheffield, Sheffield, UK

${ }^{4}$ Public Health Section, ScHARR, Regent Court, University of Sheffield, 30

Regent Street, Sheffield S1 4DA, UK

Full list of author information is available at the end of the article
} 


\section{Background}

Within the past decade, there has been a growing interest in researching the management, needs, and treatment of those with multiple long term health conditions [1]. Living with two or more chronic conditions has increasingly become more common as people age, leading to increased financial pressures on healthcare systems and treatment burden for those living with multimorbidities. The needs of these patients are complex, with those experiencing different conditions needing to attend multiple appointments with various doctors, while managing several medications [2]. This can often lead to inefficiencies in health care services, with patients attending multiple appointments, and confusion for patients who may receive conflicting advice from different specialists involved in their care [2-4]. Multimorbidity has also been found to be to be associated with increased healthcare costs, mortality rates, service use, and decreased physical functioning and quality of life $[2,3,5-7]$, highlighting the need for more research into the key determinants to detect early signs of risk factors and assess effective interventions.

Currently, multimorbidity is most commonly defined as the coexistence of two or more chronic conditions $[5,8,9]$. These conditions can range across different long-term, disorders, illnesses, and health problems [3, 8-10]. Quantifying multimorbidity proves to be challenging because there has yet to be an internationally recognised list of conditions defined as chronic [4]; one recent systematic review of observational studies on multimorbidity within primary care found that the number of conditions studied ranged from five to 335 [11]. While there has been considerable discussion around the terms multimorbidity and comorbidity (the occurrence of medical conditions additional to an index disease) $[9,12,13]$, here we focus on multimorbidity. This is based on the argument that multimorbidities may be a better construct for primary care when the focus is on the individual as a whole compared to comorbidity which may be more useful in specialist care where the emphasis is on an index disease [8].

Most research on multimorbidity is dominated by its effect on individuals and healthcare services, but the evidence base for complex interventions within primary care is very limited and little is known about patient needs [5, 14]. A recent Cochrane review found that most studies have focused on multimorbidity in older patients and evidence for interventions has been mixed, concluding that further research into particular risk factors and functional difficulties was needed [10]. There has also been little research into how patients experiencing multimorbidity view their condition, their experiences with health services and providers, and how this relates to the professional construction of multimorbidity $[5,8,11]$. Qualitative work among older patients with diabetes, depression, and osteoarthritis found that this group wanted convenient access to health care, individualised care plans, support from one coordinator of care, and continuity of relationships with health professionals [15], stressing the complex needs of those with multimorbidities.

Research into multimorbidity is fairly recent, and the epidemiology of multimorbidity is not well understood, but is required to help inform decision-making. The aim of this study was to use baseline data from the Yorkshire Health Study (YHS) to examine the patterns of multimorbidity and their association with health outcomes for residents in Yorkshire, England.

\section{Methods}

\section{Data, recruitment, sampling}

The Yorkshire Health Study is a longitudinal panel study that collects health information on adults aged 16-85. It was originally designed to address the needs of the Department of Health Healthy Weight, Healthy Lives report [16], which could not be addressed by existing population cohorts. The first wave of data was collected between 2010 and 2012 and surveyed patients residing in Yorkshire, England. Invitations to complete postal or online questionnaires were distributed to 156,866 patients (aged 16-85) recruited from 43 general medical practices of which 27,806 questionnaires were returned (15.9\% response rate). The YHS has a small undercount of younger males and those from deprived areas. Further details on the data collection methods used and how the cohort demographic profile compares to the general Yorkshire population have been published elsewhere $[17,18]$ and the second wave of data collection was completed in January 2016.

A unique design feature of the cohort is that it is the first cohort study to use the 'cohort multiple randomised controlled trial design' [19], an innovative approach to tackling some of the problems associated with pragmatic trial designs (recruitment, ethics, patient preferences and treatment comparisons). The design enables quick and easy identification of participants to multiple randomized controlled trials within the same population, facilitating easier comparability between studies. For each randomised controlled trial, those eligible are identified and a random selection offered the trial intervention; their outcomes are compared with those eligible patients not offered the intervention.

\section{Measures}

One of the original aims for the cohort was to initiate a programme of research into the management and selfmanagement of weight and long-term conditions in 
adults living in the South Yorkshire area [18]. Because of this, a wide range of information was collected allowing for detailed investigation of variations in obesity in relation to other dimensions of health, particularly chronic health conditions. Respondents were able to report on twelve 'long standing' conditions listed in the questionnaire: tiredness/fatigue, pain, insomnia, anxiety/nerves, depression, diabetes, breathing problems, high blood pressure, heart disease, osteoarthritis, stroke, and cancer. An open ended option was also provided which allowed respondents to report any other conditions not listed.

Demographic information was collected on sex, age, self-reported height, weight, and waist measurement (tape measure was provided), ethnicity, highest level of education, occupation (using 2010 National Statistics Socioeconomic Classification), and postcode information was used to measure deprivation via the 2010 Index of Multiple Deprivation (IMD). The IMD is an areabased measure for multiple deprivation for small areas in England [20]. 2010 IMD scores calculated based on 38 indicators across seven domains (e.g. income, employment, health, education) range from 0.53 to 87.80 (higher values indicating higher deprivation) and are assigned to 32,482 areas in England (also known as Lower layer Super Output Areas). IMD scores were categorised into quintile groups for our analyses. Health information related to frequency of health care usage, medication usage (non-prescription and prescription), and healthrelated quality of life using EuroQoL-5D (EQ-5D-3L) were also collected. The EuroQoL is a standardised instrument used for measuring five attributes of health status: mobility, self-care, usual activities, pain/discomfort, and anxiety/ depression [21]. The full original questionnaire and details about the distributions of these variables have been published [17, 18].

\section{Analyses}

Data was analysed using SPSS Windows v22.0. Descriptive statistics of baseline data are presented in tables, cross-tabulations, and graphical displays to illustrate patterns of multimorbidity across demographics, healthrelated quality of life, health service, and medication usage. Multimorbidity was defined as those who selfreported having at least two of the thirteen long-term health conditions (i.e. included those responding with an 'other' condition) listed in the questionnaire. Bivariate analyses were conducted to determine associations between multimorbidity and demographics, deprivation, EQ-5D scores, health service use and medication usage. A t-test was used to analyse differences in mean number of long term health conditions across gender and ethnicity and one-way ANOVA was used for differences across age groups, deprivation quintile groups, and body mass index (BMI) groups. Chi-squared tests were used to measure differences in the prevalence of multimorbidity between all demographic variables $(\mathrm{p}<0.05)$. Sample weights were applied to data to adjust for groups who were over-represented in the cohort compared to the general adult Yorkshire population (females, those who are older and those living in less deprived areas) $[17,22]$. With the exception of Table 1 , data presented here are based on the weighted sample.

\section{Results}

Table 1 presents sample characteristics, mean number of long standing conditions, and proportions of those with multimorbidity. Within the unweighted sample, slightly

Table 1 Unweighted sample characteristics and long standing health conditions for Yorkshire Health Study Wave $1(N=27,806)$

$\mathrm{n}(\%) \quad$ Mean number of 2 or more conditions $\mathrm{c}^{\mathrm{c}}$ conditions $\left(\mathrm{SD}^{\mathrm{a}}\right)^{\mathrm{b}} \mathrm{n}(\%)$

\begin{tabular}{llll}
\hline $\begin{array}{c}\text { Gender } \\
\text { Male }\end{array}$ & $12155(43.7)$ & $1.50(1.78)$ & $4551(37.4)$ \\
Female & $15651(56.3)$ & $1.49(1.80)$ & $5781(36.9)$ \\
Age Group & & & \\
$\leq 24$ & $1734(6.2)$ & $0.47(0.92)$ & $188(10.8)$ \\
$25-34$ & $2639(9.5)$ & $0.63(1.15)$ & $391(14.8)$ \\
$35-44$ & $3516(12.6)$ & $0.83(1.35)$ & $708(20.1)$ \\
$45-54$ & $4489(16.1)$ & $1.19(1.62)$ & $1302(29.0)$ \\
$55-64$ & $5938(21.4)$ & $1.63(1.80)$ & $2412(40.6)$ \\
$65-74$ & $5827(21.0)$ & $2.09(1.93)$ & $3068(52.7)$ \\
$\geq 75$ & $3254(11.7)$ & $2.53(1.93)$ & $2125(65.3)$
\end{tabular}

Ethnicity

White $\quad 26419(95.0) \quad 1.51(1.80) \quad 9961(37.7)$

Non-White $1095(3.9) \quad 1.05(1.61) \quad 278(25.4)$

Deprivation Quintile

Least Deprived $3948(14.2) \quad 1.08(1.46) \quad 1060(26.8)$

Q2 $\quad 6923(24.9) \quad 1.30(1.62) \quad 2275(32.9)$

Q3 $\quad 4602(16.6) \quad 1.39(1.69) \quad 1615(35.1)$

Q4 $\quad 5116(18.4) \quad 1.62(1.85) \quad 2098(41.0)$

Most Deprived $7142(25.7) \quad 1.88(2.03) \quad 3263(45.7)$

Body Mass Index ${ }^{d}$

$\begin{array}{llll}\text { Underweight } & 436(1.6) & 1.29(1.88) & 126(28.9) \\ \text { Normal } & 11101(39.9) & 1.10(1.51) & 3040(27.4) \\ \text { Overweight } & 9671(34.8) & 1.52(1.74) & 3772(39.0) \\ \text { Obese } & 5190(18.7) & 2.26(2.10) & 2833(54.6)\end{array}$

Missing data not shown in table

a Standard deviation

${ }^{\mathrm{b}}$ Differences between means within ethnicity, age-group, deprivation and BMI group differed significantly $\mathrm{p}<0.001$ (t test for independent samples for ethnicity; one-way ANOVA for age-group, deprivation, and BMI group)

${ }^{c}$ Differences between categories within age, ethnicity, deprivation, and BMI group differed significantly $\mathrm{p}<.0001$ ( $\mathrm{x}^{2}$ test for $2 \times \mathrm{n}$ tables)

${ }^{d}$ Calculated by self-reported weight $(\mathrm{kg})$ divided by height-squared $\left(\mathrm{m}^{2}\right)$. Categories are based on WHO cut-offs 
over one-third ( $n=10,332)$ had experienced multimorbidity (reported having two or more long-standing conditions). This estimate lowered to $31 \%$ when based on weighted data (data not shown). The mean number of self-reported long-term health conditions varied significantly depending on age, ethnicity, index of multiple deprivation (IMD) quintile and BMI group. Multimorbidity was higher among those who were older, of white ethnicity, resided in more deprived areas and were overweight or obese. Overall, $65 \%$ of those aged $75+, 38 \%$ of white participants, and $55 \%$ of obese participants reported having at least two long-term health conditions. These demographic associations remained statistically significant when analyses were conducted on weighted data (results not presented), and also indicated a significant difference in multimorbidity between males and females ( $29 \%$ vs. $32 \%$ respectively). Within the cohort, there was a clear social gradient between deprivation and multimorbidity. Nearly half (46\%) of those living in the most deprived areas experienced multimorbidity compared to $27 \%$ of those living in the least deprived areas (Table 1). This link between high deprivation and multimorbidity was consistent across all age groups, with prevalence reaching the highest for those aged over 80 years and living in the most deprived areas (over 70 \%) (Fig. 1).

Of those who reported any long term-conditions $(n=16,760), 38.4 \%(n=6428)$ reported experiencing only one condition. Those who reported a stroke $(n$ $=400$ ) reported experiencing more multiple longterm limiting conditions, with $50 \%$ reporting experiencing four or more additional chronic conditions
(Fig. 2). Insomnia was the second highest condition with $46 \%$ of those experiencing this condition along with at least four others. With the exception of 'other' conditions, the condition with the least frequently reported number of conditions alongside it was breathing problems, $31 \%$ of these participants only experienced it alone. Figure 3 specifies the proportion of accompanying conditions alongside each condition. Roughly half of those who had reported stroke also reported experiencing fatigue, chronic pain, and high blood pressure. Osteoarthritis and pain was the most frequent combination of two conditions (66\% of those with arthritis reported also having pain), followed by insomnia and fatigue (65 \% of those with insomnia reported fatigue). Fatigue and pain were commonly reported among other long-term health conditions (Fig. 3).

Within the cohort there was a negative relationship between number of self-reported long-term conditions and self-reported quality of life (Table 2); Participants with five or more conditions reported a mean EQ-5D score of 0.355 (range $-0.594-1, \mathrm{SE}=0.008$ ), nearly three times lower than the mean score for participants with no conditions 0.945 (range -0.594- $1, \mathrm{SE}=0.001$ ). Multimorbidity was positively associated with more health service visits (Table 3) and medication use (Fig. 4). Participants with no long term health conditions reported taking on average 1.81 medications (non-prescription and prescription) compared to those with at least two long-term conditions who reported 3.8 medications and those with $5+$ conditions who reported 7.5 medications on average.

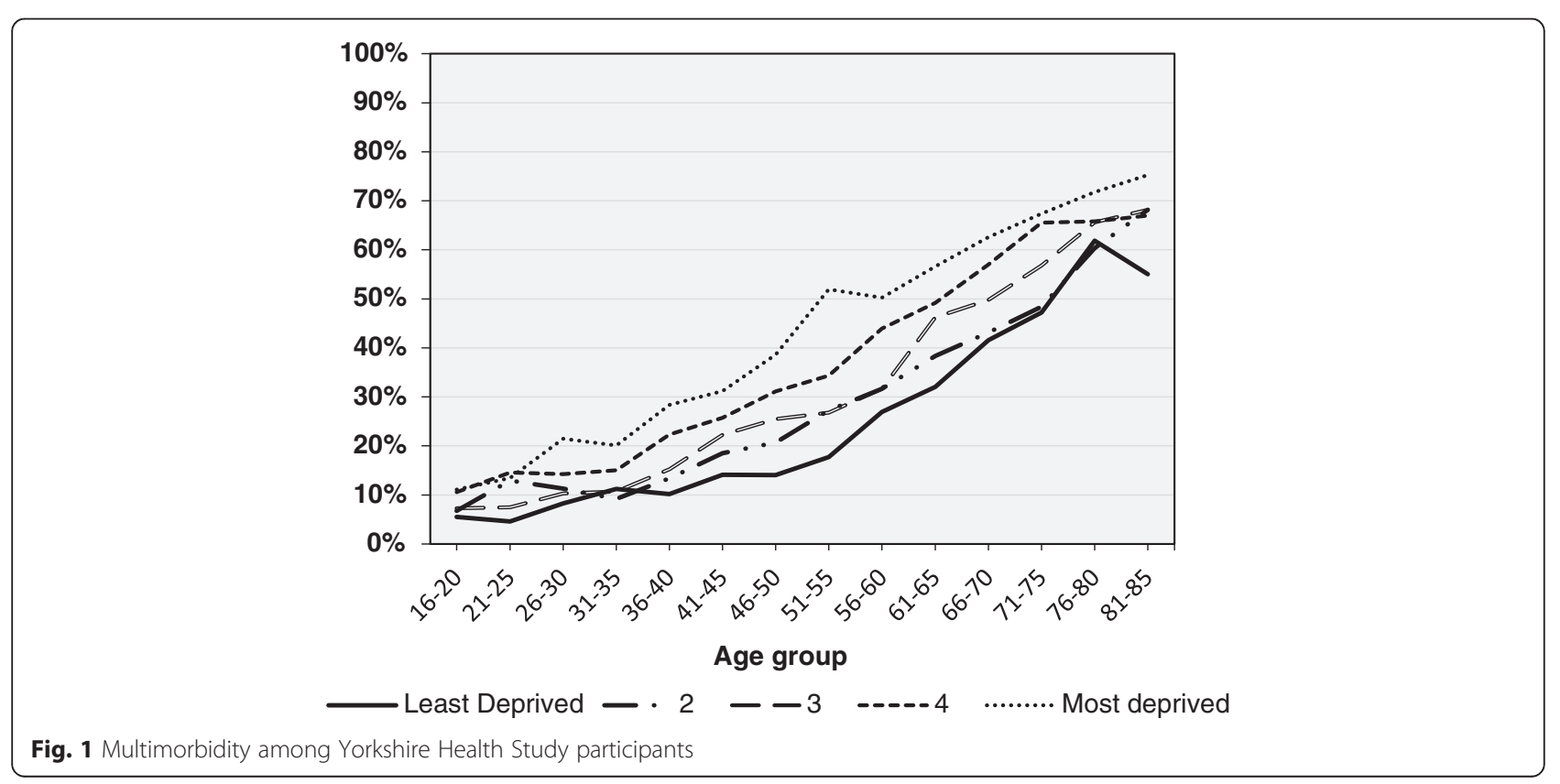




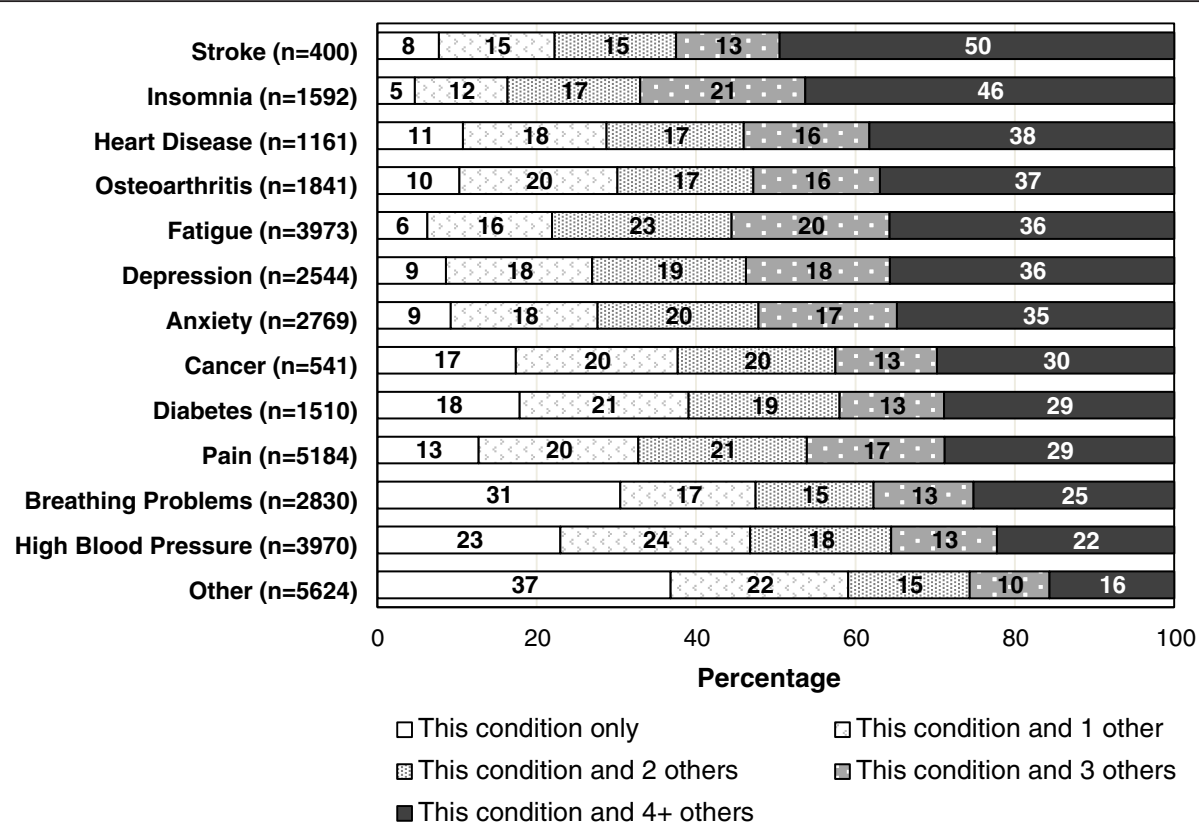

Fig. 2 Distribution of long-standing conditions reported by Yorkshire Health Study participants

\section{Discussion}

Research into multimorbidity has been steadily increasing over the years. Therefore a standard definition of multimorbidity is required if research in this area is to advance. The level of prevalence of multimorbidity can vary across studies depending on the measure used (e.g. prevalence estimates have ranged from 13 to $95 \%$ across primary care studies in Europe, North America and Australia [11]). Conditions within multimorbidity vary from study to study $[5,11,23]$. For instance, some might classify depression and anxiety separately while others could argue that because they are clinically closely interrelated they should not be treated as separate entities when classifying those with multimorbidity $[6,8]$. In this study we chose to examine depression and anxiety as separate entities when estimating multimorbidity as has been done elsewhere [3]. Based on a simple multimorbidity definition (the co-occurrence of two or more conditions whether coincidental or not [12]), overall we found that $37 \%$ of participants sampled in the Yorkshire Health Study had experienced multimorbidity. This lies in between other UK estimates of multimorbidity that have ranged from $23 \%$ [3] and $30 \%$ in Scotland [24] to $58 \%$ in England [4]. However it is important to bear in mind that each study used a different range of long-term conditions when estimating multimorbidity (e.g. learning disability, thyroid disease, epilepsy, etc.). Within the weighted sample, females were also more likely to report experiencing multimorbidity, consistent with other UK, European, and American studies [1, 11, 25]. Consistent with trends [3], multimorbidity steadily increased with age in the YHS cohort. A clear link between multimorbidity and deprivation within the YHS cohort was also identified, supporting evidence found elsewhere which indicate higher levels of multimorbidity among lower socioeconomic groups $[3,4]$.

Regarding health outcomes associated with multimorbidity, our findings suggest that multimorbidity is associated with poor health-related quality of life (measured through EQ-5D scores), which is consistent with findings from previous studies that have used other healthrelated quality of life questionnaires (such as the SF-36) [6]. Patterns of increasing recent hospital, GP, nurse, dietician, physiotherapist, alternative therapist, and other carer visits among those with more long-term conditions were also identified. Health service use in future waves of the YHS can be compared to multimorbid participants' baseline service use and used to prioritize and adapt the commissioning and management of future services, particularly by identification of need in different populations and locations (e.g. areas of high deprivation). Guidance on the decisions for multiple medication use are currently lacking within the United Kingdom [26] and findings from our study suggest that while the proportion of non-prescription and prescription medications taken is fairly consistent across the number of conditions reported, among YHS participants, the number of current medications increases substantially to an average of 7 medications for those with five or more self-reported long-term health 


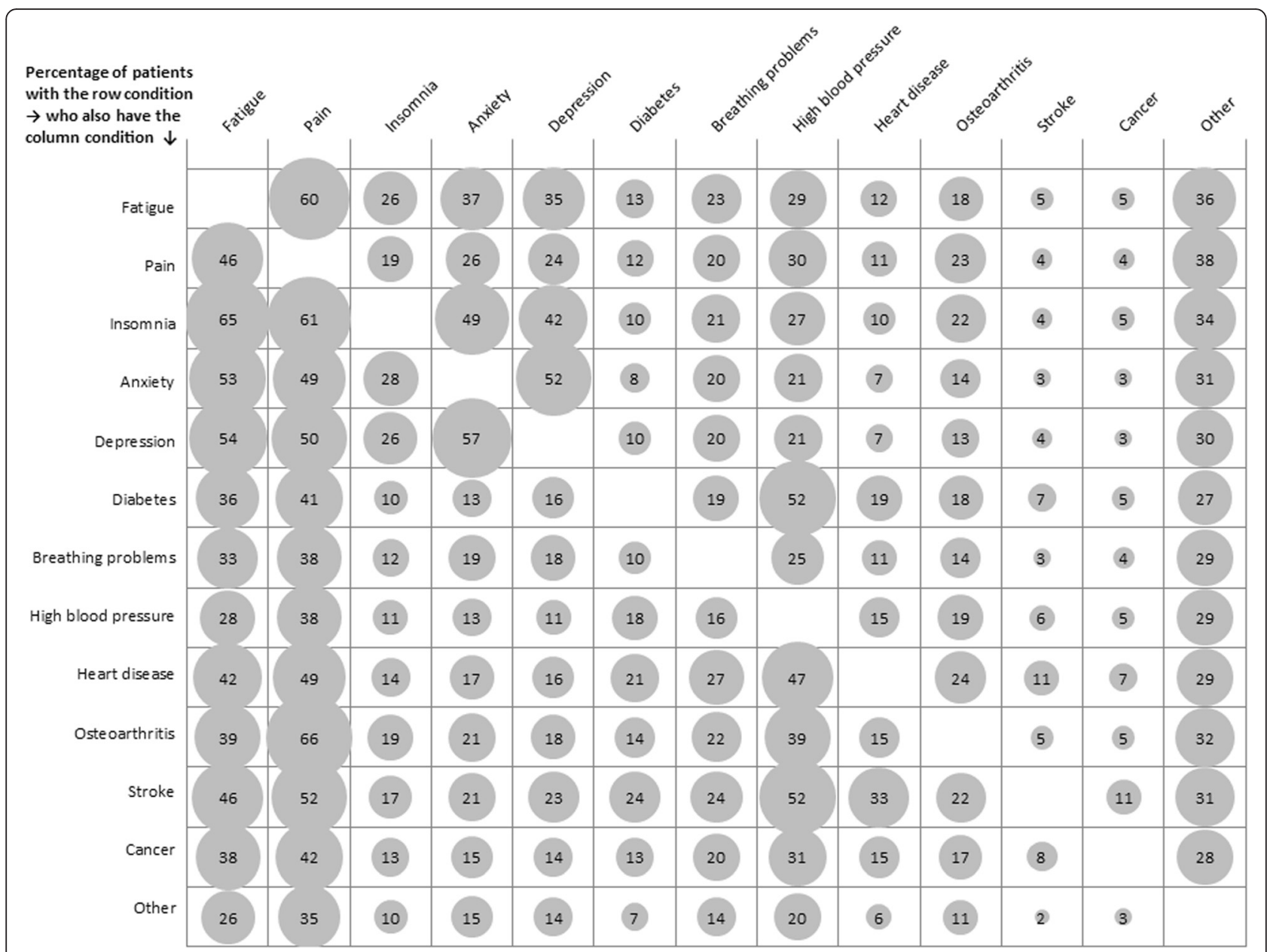

Fig. 3 Self-reported long-term health conditions among Yorkshire Health Study participants

conditions. Furthermore data from our survey supports the link between obesity and multimorbidity which has been found elsewhere [27]. Information on a number of weight management strategies within the YHS [22] could be used to further explore this link and determine whether weight loss and strategies used would be associated with a reduction in the number of chronic conditions over time.
Table 2 EQ-5D by number of long-term conditions

\begin{tabular}{lllll}
\hline $\begin{array}{l}\text { Number of } \\
\text { conditions }\end{array}$ & Mean $^{\mathrm{a}}$ & Standard error & $95 \%$ confidence interval & $\mathrm{N}$ \\
\hline 0 & 0.945 & 0.001 & $0.943-0.947$ & 12978 \\
1 & 0.859 & 0.002 & $0.854-0.863$ & 6012 \\
2 & 0.754 & 0.004 & $0.747-0.762$ & 3279 \\
3 & 0.651 & 0.006 & $0.640-0.662$ & 2083 \\
4 & 0.532 & 0.008 & $0.516-0.549$ & 1297 \\
$5+$ & 0.355 & 0.008 & $0.339-0.371$ & 1677 \\
\hline
\end{tabular}

${ }^{a}$ Range $=1$ (perfect health) through to 0 (dead) to -0.594 (worse than dead) [31] EQ-5D score not available for all participants
Some limitations of our data should be considered. Data within the YHS cohort is based on self-report, which is prone to known biases [28]. However, with regard to multimorbidity, a benefit of using self-report data is that it includes conditions that patients may not always report to clinicians or clinicians may underrecord (e.g. anxiety or pain) [3]. Another limitation is

Table 3 Healthcare use in last three months by long-term conditions (mean number of appointments, standard error)

\begin{tabular}{lllll}
\hline Number of LTCs & Inpatients & Outpatients & Day cases & A\&E $^{\mathrm{b}}$ \\
\hline 0 & $0.11(0.010)$ & $0.26(0.011)$ & $0.07(0.009)$ & $0.11(0.005)$ \\
1 & $0.29(0.041)$ & $0.72(0.031)$ & $0.16(0.015)$ & $0.16(0.009)$ \\
2 & $0.52(0.063)$ & $1.09(0.049)$ & $0.33(0.051)$ & $0.23(0.015)$ \\
3 & $1.10(0.218)$ & $1.35(0.073)$ & $0.44(0.073)$ & $0.35(0.034)$ \\
4 & $1.13(0.190)$ & $1.76(0.127)$ & $0.49(0.070)$ & $0.46(0.053)$ \\
$5+$ & $2.05(0.278)$ & $2.51(0.138)$ & $1.10(0.171)$ & $0.59(0.049)$
\end{tabular}

a LTCs = Long-term conditions

${ }^{\mathrm{b}}$ Accident and Emergency 


\section{Number of medications by long-term conditions}

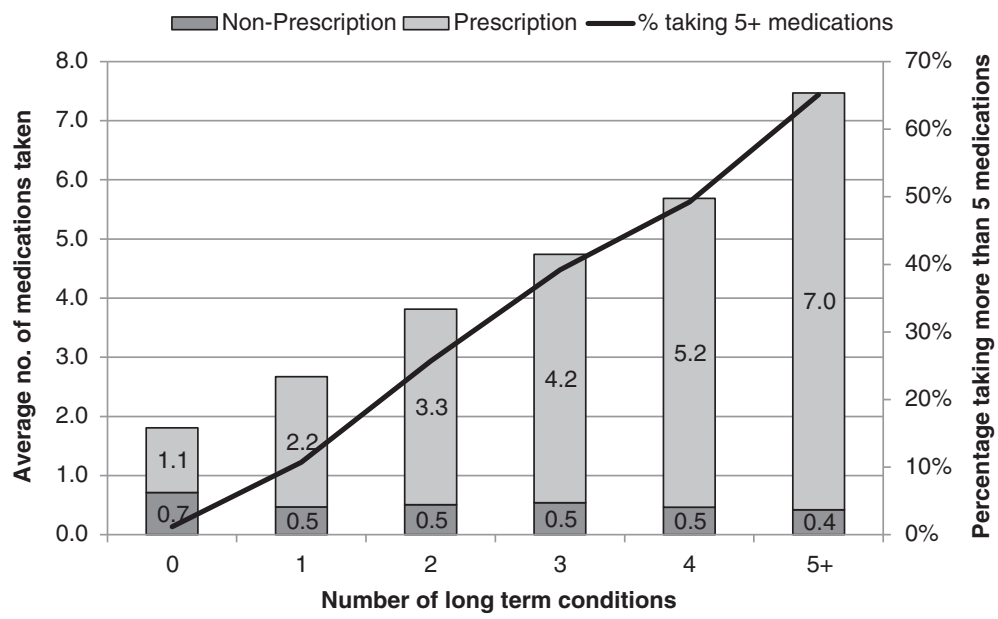

Fig. 4 Yorkshire Health Study medication use by long-term conditions

that those who responded with an 'other' condition could have also responded with multiple conditions in the free text response (e.g. arthritis, hypothyroidism, high cholesterol, epilepsy, glaucoma, irritable bowel syndrome, etc.). Within this study we have not analysed these free text conditions, therefore the number of conditions for this category may be under or over estimated. Finally, though cross-sectional designs can provide prevalence estimates and descriptive information that would allow for identification of potential risk factors, they do not allow for a deeper understanding of patients' experiences of living with multimorbidities over the life course and how these needs and experiences change as new morbidities are acquired over time. Because of this, prospective cohort studies have been regarded as the 'gold standard' for studying multimorbidity [5]. One recent systematic review of six prospective cohort studies within primary care found that these studies mainly focused on only healthcare utilisation, patient's physical functioning, and risk factors and concluded that future cohort studies should also consider examining longitudinal links between socioeconomic factors and mental illness [5].

The National Institute for Health Care and Excellence (NICE) is currently consulting on clinical practice guidance for the management of multimorbidity, aiming to publish guidelines in 2016 [29]. Studies have indicated that more research should investigate modifiable risk factors (e.g. smoking and diet), socio-economic disparities in multimorbidity, and seek to examine multimorbidity longitudinally through large-scale prospective designs $[5,11]$. Though not examined in this study, the second wave of data collection (2013-2015) for the YHS which was recently completed in January 2016 could provide further insight into addressing socioeconomic patterns of multimorbidity over the life course or other issues e.g. determining the average onset age for multimorbidity, identifying risk factors associated with multimorbidity over time (via health behaviours collected in the questionnaire but not examined in this study e.g. alcohol consumption, diet, and exercise), or assessing potential complex interventions within primary settings for patients experiencing multimorbidity and examining the cost-effectiveness of those interventions (through quality of life or life satisfaction measures). Because the YHS was designed to provide a cohort facility for multiple trials and other studies, its recruitment would allow researchers to examine specific subgroups (e.g. demographic) or groups that may be particularly vulnerable to multimorbidity (e.g. certain ethnic groups, patients experiencing specific conditions). The majority of the cohort (approximately $78 \%$ ) provided consent for researchers to access their health records [17] which would also enable data linkage with other disease diagnoses or healthcare and medication usage reported in other records. An ongoing example is a study that links YHS data with data from cancer screening programmes to examine the impact of morbidities (and hence multimorbidity) on the uptake of colorectal cancer screening [30].

\section{Conclusions}

Within our baseline, our findings support evidence around poor health outcomes for those with multimorbidity and indicate a clear socio-economic patterning of multimorbidity, with those living in the most depriving areas being more likely to experience multimorbidities. Overall the YHS is a useful resource for those who are interested in chronic disease and multimorbidity research 
and would provide researchers with an efficient way of recruiting patients from a population-based cohort for a variety of different studies (e.g. randomised control trials, qualitative, cross-sectional, or longitudinal) alongside a dataset of rich information on a wide range of relevant health-related behaviours and conditions for analysing patterns and trends in chronic disease over time.

\section{Abbreviations}

BMI, Body Mass Index; GP, General Practitioner; NHS, National Health Service; YHS, Yorkshire Health Study

\section{Acknowledgements}

We are grateful to all the individuals who have enrolled in the cohort. We also acknowledge the GP practice staff for their contribution in the recruiting process. This publication is the work of the authors and does not necessarily reflect the views of the Yorkshire Health Study Management Team or Steering Committee. This paper presents independent research by the National Institute for Health Research Collaboration for Leadership in Applied Health Research and Care Yorkshire and Humber (NIHR CLAHRC YH, http://clahrc-yh.nihr.ac.uk). The views and opinions expressed are those of the authors, and not necessarily those of the NHS, the NIHR or the Department of Health.

\section{Funding}

This work was supported by the National Institute for Health Research (NIHR) Collaborations for Leadership in Applied Health Research and Care (CLAHRC) for South Yorkshire (Obesity Theme) and the University of Sheffield. This report is independent research supported (in part) by the National Institute for Health Research (Mark Strong - Post-Doctoral Fellowship, PDF-2012-05-258). The views expressed in this publication are those of the author(s) and not necessarily those of the NHS, the National Institute for Health Research or the Department of Health.

\section{Availability of data and materials}

The data are managed by the University of Sheffield's Clinical Trials Research Unit. Anonymized data and details regarding using the resource for recruiting participants to studies can be gathered by contacting Clare Relton (c.relton@sheffield.ac.uk). Multi-disciplinary collaboration is strongly encouraged.

\section{Authors' contributions}

CR conceived of the idea for the article and $J \mathrm{~L}$ and BK helped to focus its research question. $M G, J L$, and $B K$ conducted the data analyses. JL wrote the first working draft and $M G, B K, E H, C S, A H, C C, M S$, and $C R$ were involved in revising on later drafts and gave final approval of the version to be published.

\section{Competing interests}

The authors declare that they have no competing interests.

\section{Ethics approval and consent to participate}

Ethical approval was obtained from the Leeds East National Health Service (NHS) Research Ethics Committee (ref: 09/H1306/97). An information sheet was provided to participants and consent to participate in the YHS study was implied if a questionnaire was returned to the research team.

\section{Author details}

${ }^{1}$ School of Health and Related Research (SCHARR), University of Sheffield, Sheffield, UK. ²Department of Geography \& Planning, University of Liverpool, Liverpool, UK. ${ }^{3}$ Barnsley Hospital NHS Foundation Trust, Barnsley, UK. ${ }^{4}$ Public Health Section, ScHARR, Regent Court, University of Sheffield, 30 Regent Street, Sheffield S1 4DA, UK.

\section{Received: 27 February 2016 Accepted: 21 July 2016}

Published online: 27 July 2016

\section{References}

1. Marengoni A, Angleman S, Melis R, Mangialasche F, Karp A, Garmen A, Meinow B, Fratiglioni L. Aging with multimorbidity: a systematic review of the literature. Ageing Res Rev. 2011;10(4):430-9.
2. Wallace E, Salisbury C, Guthrie B, Lewis C, Fahey T, Smith SM. Managing patients with multimorbidity in primary care. BMJ. 2015;350:h176.

3. Barnett K, Mercer SW, Norbury M, Watt G, Wyke S, Guthrie B. Epidemiology of multimorbidity and implications for health care, research, and medical education: a cross-sectional study. Lancet. 2012;380(9836):37-43.

4. Salisbury C, Johnson L, Purdy S, Valderas JM, Montgomery AA. Epidemiology and impact of multimorbidity in primary care: a retrospective cohort study. $\mathrm{Br} \mathrm{J}$ Gen Pract. 2011;61(582):e12-21.

5. France EF, Wyke S, Gunn JM, Mair FS, McLean G, Mercer SW. Multimorbidity in primary care: a systematic review of prospective cohort studies. $\mathrm{Br} J \mathrm{Gen}$ Pract. 2012;62(597):e297-307.

6. Fortin M, Lapointe L, Hudon C, Vanasse A, Ntetu AL, Maltais D. Multimorbidity and quality of life in primary care: a systematic review. Health Qual Life Outcomes. 2004;2:51

7. Naylor C, Parsonage M, McDaid D, Knapp M, Fossey M, Galea A: Long-term conditions and mental health: the cost of co-morbidities. London: The King's Fund; 2012.

8. Valderas JM, Starfield B, Sibbald B, Salisbury C, Roland M. Defining comorbidity: implications for understanding health and health services. Ann Fam Med. 2009;7(4):357-63.

9. Mercer SW, Smith SM, Wyke S, O'Dowd T, Watt GC. Multimorbidity in primary care: developing the research agenda. Fam Pract. 2009;26(2):79-80.

10. Smith SM, Soubhi H, Fortin M, Hudon C, O'Dowd T. Interventions for improving outcomes in patients with multimorbidity in primary care and community settings. Cochrane Database Syst Rev. 2012;4:CD006560.

11. Violan C, Foguet-Boreu Q, Flores-Mateo G, Salisbury C, Blom J, Freitag M, Glynn L, Muth C, Valderas JM. Prevalence, determinants and patterns of multimorbidity in primary care: a systematic review of observational studies. PLoS One. 2014;9(7), e102149.

12. van den Akker M, Buntinx F, Knottnerus JA. Comorbidity or multimorbidity. Eur J Gen Pract. 1996;2(2):65-70.

13. van den Akker M, Buntinx F, Roos S, Knottnerus JA. Problems in determining occurrence rates of multimorbidity. J Clin Epidemiol. 2001;54(7):675-9.

14. Mercer SW, Gunn J, Wyke S: Improving the health of people with multimorbidity: the need for prospective cohort studies. Journal of Comorbidity. 2011;1(1):4.

15. Bayliss EA, Edwards AE, Steiner JF, Main DS. Processes of care desired by elderly patients with multimorbidities. Fam Pract. 2008;25(4):287-93.

16. Department of Health: Health Weight, Healthy Lives: A Cross-Government Research and Surveillance Plan. In. London: Department of Health; 2008.

17. Green MA, Li J, Relton C, Strong M, Kearns B, Wu M, Bissell P, Blackburn J, Cooper C, Goyder E, et al. Cohort profile: The Yorkshire Health Study. Int J Epidemiol. 2014

18. Relton C, Bissell P, Smith C, Blackburn J, Cooper CL, Nicholl J, Tod A, Copeland R, Loban A, Chater T, et al. South Yorkshire Cohort: a 'cohort trials facility' study of health and weight - protocol for the recruitment phase. BMC Public Health. 2011;11:640.

19. Relton C, Torgerson D, O'Cathain A, Nicholl J: Rethinking pragmatic randomised controlled trials: introducing the "cohort multiple randomised controlled trial" design. BMJ. 2010; 340:c1066.

20. Government DfCaL: English Indices of Deprivation 2010: Guidance Document. In. https://www.gov.uk/government/uploads/system/uploads/attachment data/file/6222/1871538.pdf; 2011: 4. Accessed 25 July 2016.

21. Rabin R, de Charro F. EQ-5D: a measure of health status from the EuroQol Group. Ann Med. 2001;33(5):337-43.

22. Relton C, Li J, Strong M, Holdsworth M, Cooper R, Green M, Bissell P. Deprivation, clubs and drugs: results of a UK regional population-based cross-sectional study of weight management strategies. BMC Public Health. 2014;14:444.

23. Huntley AL, Johnson R, Purdy S, Valderas JM, Salisbury C. Measures of multimorbidity and morbidity burden for use in primary care and community settings: a systematic review and guide. Ann Fam Med. 2012;10(2):134-41.

24. Macleod U, Mitchell E, Black M, Spence G. Comorbidity and socioeconomic deprivation: an observational study of the prevalence of comorbidity in general practice. Eur J Gen Pract. 2004;10(1):24-6.

25. Agborsangaya CB, Lau D, Lahtinen M, Cooke T, Johnson JA. Multimorbidity prevalence and patterns across socioeconomic determinants: a crosssectional survey. BMC Public Health. 2012;12:201.

26. National Institute for Health and Care Excellence Scope [https://www.nice org.uk/guidance/gid-cgwave0704/resources/multimorbidity-final-scope2]. Accessed 25 July 2016

27. Agborsangaya CB, Majumdar SR, Sharma AM, Gregg EW, Padwal RS. Multimorbidity in a prospective cohort: prevalence and associations 
with weight loss and health status in severely obese patients. Obesity. 2015;23(3):707-12.

28. Lydeard S. The questionnaire as a research tool. Fam Pract. 1991;8(1):84-91.

29. Multimorbidity: clinical assessment and management [http://www.nice.org. uk/guidance/indevelopment/gid-cgwave0704]. Accessed 25 July 2016.

30. Exploratory Analysis on the Impact of Morbidities on Colorectal Cancer Screening Uptake [https://clinicaltrials.gov/ct2/show/study/NCT02503969]. Accessed 25 July 2016.

31. Kearns B, Ara R, Young T, Relton C. Association between body mass index and health-related quality of life, and the impact of self-reported long-term conditions - cross-sectional study from the south Yorkshire cohort dataset. BMC Public Health. 2013;13:1009.

Submit your next manuscript to BioMed Central and we will help you at every step:

- We accept pre-submission inquiries

- Our selector tool helps you to find the most relevant journal

- We provide round the clock customer support

- Convenient online submission

- Thorough peer review

- Inclusion in PubMed and all major indexing services

- Maximum visibility for your research

Submit your manuscript at www.biomedcentral.com/submit
Biomed Central 